\title{
ON THE USE OF OPERATIONS RESEARCH MODELS FOR MAINTENANCE DECISION MAKING
}

\author{
Rommert Dekker'
}

\begin{abstract}
In this paper we analyse the role of operations research models in maintenance decision making. After an overview of these models and their applications, we investigate the bottlenecks for application. Successively we discuss decision support systems and the problems concerning data. From an analysis of present trends we conclude not only that more applications can be expected, but that also more application oriented research needs to be done.
\end{abstract}

\section{Introduction}

First operations research models for maintenance optimisation were developed in the sixties by pioneers like Barlow, Proschan, Jorgenson and McCall. Basically, a maintenance optimisation model is a mathematical model in which both costs and benefits of maintenance are quantified and in which an optimum balance between both is obtained. The area proved to be fruitful for researchers. Reviews were made regularly: Sherif (1982) reports on 818 articles and many more have appeared since.

Regular applications of these models were made only some twenty years later when PC-based decision support systems (dss) brought them to the maintenance manager (Dekker (1992a)). In this paper we focus on the bottlenecks encountered in the use of such systems. The analysis is based on personal experience with developing such a system and evaluating its applications as well as on a literature review of applications. The structure of this paper is as follows. Maintenance

1 Erasmus University Rotterdam, Econometric Institute, P.O. Box 1738, 3000 DR Rotterdam, The Netherlands 
optimisation models are introduced in section 2. In section 3 we present a review of applications and discuss decison support systems in section 4. Section 5 deals with data problems. Finally, future prospects and conclusions are stated in sections 6 and 7.

\section{Maintenance optimisation models}

In general, maintenance optimisation models cover four aspects: (i) a description of a technical system, its function and its importance, (ii) a modelling of the deterioration of the system in time and possible consequences for the system, (iii) a description of the available information about the system and the actions open to management and (iv) an objective function and an optimisation technique which helps in finding the best balance. The results of these models are the following. First of all, policies can be evaluated and compared with respect to cost effectiveness and reliability characteristics. E.g. a condition monitoring policy can be compared with a planned preventive maintenance strategy. Secondly, models can assist in the timing aspect: how often to inspect or to maintain, e.g. once or twice a year. Finally, models can also be of help in determining effective and efficient schedules and plans, taking all kind of constraints into account.

\section{Applications}

\subsection{An overview}

Dekker (1992a) provides an overview of applications which have been published in open literature. The total of 89 papers on applications which were found may seem little, but only a small part of all applications is likely to be published. Most case studies were written in cooperation with academic reliability researchers (47 out of the 89). Few originate from industry and if they do, often from an industrial research institute. Consultants were badly represented, which gives the impression that, 
until recently, application of maintenance optimisation models was only possible by the people who developed them. This situation has changed with the emergence of commercial decision support systems for maintenance optimisation. What strikes is that many papers describe a tailored-made model for the problem at hand. Apparently one often has to include problem specific factors.

There are several areas and industries in which the models have been applied; popular are equipment and vehicle replacements, inspection optimisation, road maintenance and scheduling of maintenance of electric power stations.

Equipment overhauls comprise the largest group of applications. Another fruitful area is vehicle replacement, concerning buses, fork lift trucks, ambulances etc. One advantage in case of vehicles, is that the large number of units allows for data pooling. For equipment this is not always possible as similar equipment in different conditions may have quite a different failure pattern. A third and promising area for optimisation is road maintenance. Here, a main problem is the allocation of budgets to various projects. The first successful case study was presented in Golabi (1982) (he quoted savings of millions of dollars!). Later quite some applications appeared in the US. Other countries followed, including the Netherlands. One reason behind the successes in this area, is that a lot of data is collected in standard ways on deterioration. Hence the need arises to make use of this data in decision making. Furthermore, there is less time pressure on decision making compared to other maintenance organisations, and as funds have to be allocated between various projects in an objective and defendable way, there is a need for objective methods, hence also for operations research models. Related applications concern water resources infrastructure, pipelines and railway track. A fourth application area concerns the scheduling of 
electric power stations overhauls. The overview from Kralj and Petrovic (1987) gives the impression that several operations research methods have been applied.

The problems encountered in applying maintenance optimisation models mentioned in the case studies frequently concern data collection and analysis. As most papers advocate a technique, negative remarks on maintenance optimisation are hard to find. An exception is Van Bommel and Roes (1986), who describe a failure to apply standard models and therefore (successfully) resort to failure analysis.

\subsection{Software packages for maintenance optimisation}

First packages for maintenance optimisation were running on micro computers, like the HP 9845. They were developed by academics with interest in practical applications. Later, the personal computer became a popular platform for development of maintenance optimisation software. A successful example is the MAINOPT package (see Woodhouse (1986)) in Europe; examples in the US are the AGE/CON, PERDEC and RELCODE packages, all including analytical models. MAINOPT is in fact the first package which does not originate from researchers, a fact which does show that theory has been picked up by others.

\subsection{Maintenance optimisation in the Netherlands}

Although MAINOPT was developed in the UK, it also obtained successes in the Netherlands, especially at Shell Pernis Refinery (see Dekker et al. (1993)). In more than twenty projects it was used and the savings run in the millions. The Koninklijke/Shell-Laboratorium, Amsterdam has been active in developing decision support systems for Shell use only. A simulation package was also developed at Eindhoven University of Technology (see Both (1989), initially on a mini computer and later 
converted to a PC, but it is not commercial. The KEMA has been active and now supplies the KMOSS package, containing four different models (Van Gestel (1992)). The Dutch State Water Authority, RWS (Rijkswaterstaat) had a prototype decision support system developed for road maintenance by Worms and Van Harten (1992). The FEL-TNO laboratory developed a package for maintenance of civil structures, using Markov decision models (see Hontelez (1992)). Finally, the Delft Hydraulics Laboratory did some cases on maintenance optimisation for the RWS as well. Almost all packages deal with strategic maintenance problems.

\section{Decision support systems for maintenance optimisation}

It will be obvious that academics and some consultants have the knowledge to apply maintenance optimisation models. Yet not many applications will come off the ground, unless the knowledge and technology needed to do applications can be transferred to a maintenance engineer. It is here that information technology plays an important role. We will analyse what role it plays and distinguish four aspects.

(i) computers are necessary to do the calculations

Almost no maintenance optimisation model yields results which require hand calculations only. Except for some specific cases for which graphical methods have been developed or explicit solutions exist, one has to apply numerical integration or to solve sets of equations to determine the optimal policy. In most cases PC's can do the job. The main bottleneck lies in a userfriendly interface. The PC is and presumably will remain the right platform, certainly for the next decade. The interface with maintenance management information systems is not crucial, as optimisation models tend to use other data than is stored in these systems (see also section 5). Only if optimisation routines are used for regular scheduling, a link is necessary. 
(ii) the importance of user interfaces and userfriendly computer systems Although it is not that difficult to write a software program for many of the optimisation models, it is very difficult to have it used by others to solve their problems. Just combining a number of models, algorithms and a graphical interface into a program is not sufficient. Models are, by definition, abstractions and simplifications from reality. If problems are repetitive, such as in road maintenance, one has the time to find the right model. If they are nonrepetitive, a dss should guide the maintenance engineer in the modelling, allowing various options. Not too many because this may confuse him or her. Hence a general problem structuring should be made on forehand which should fit on the bulk of the problems. That is what developing a dss for maintenance optimisation makes time consuming and therefore costly. A problem in this respect is that concepts like failure, repair can have several interpretations, and it is important to state and explain the assumptions used in the models. People will only use software packages if they can easily communicate with them and understand what they are doing (in the end they should be able to convince their managers of the results). This asks for very friendly userinterfaces, in which data can be provided in the way they are available to the user and in which results can be shown in the way the user wants. Today, there is no package on the market which really meets all these requirements. Finally, there is always a learning step in using software systems. If they are not regularly used, there is a serious danger that the experience needed to run them, fades away, with the result that they are put aside. Courses are often provided with packages, but in practice this has shown to be insufficient.

(iii) there is a lack of general modelling knowledge

There is a large variety of systems requiring maintenance and although numerous models have been published, there is insufficient knowledge about how standard models can be used for a given problem. Building a 
dss for each company is still costly, so it is important to know which models are suited for which problems. Models for civil structures, like roads, dams and buildings, tend to be driven by norms for functioning, rather than by costs of nonfunctioning. This makes them different from models for production equipment. More research needs to be done to arrive at a set of standard models for a number of well-described problem areas. A first attempt is given in Dekker (1992b).

(iv) the use of decision support systems in any organisation requires a change of culture

Such a change is difficult to achieve especially in a maintenance organisation, as it is that focused on technical problem solving under time constraints. Maintenance management has to accept and demand quantitative methods as basis for decision making. They will only do so, if they have confidence in them. A focal point in the maintenance organisation, familiar with operations research and computers, can be vital for the success of decision support systems. However, one should realise that use of quantitative techniques is not synonymous with taking the right decisions. Technical knowledge will remain very important.

\section{Data}

An essential part of a maintenance optimisation model is the modelling of the deterioration and the occurrence of failures of a system in time. Maintenance actions will only be effective and efficient if they specifically address the relevant deterioration and failure mechanisms. Global statistics, like an average failure rate, are therefore inappropriate for most maintenance actions. Analysing data without knowing the underlying failure mechanisms can lead to totally wrong results (consider e.g. failures caused by wearout and by mal operation). This is often aggrevated by an inadequate system-component structuring which is used in maintenance reporting and the fact that malfunctioning of one 
part may induce failures in others. Maintenance information systems thus mainly store accounting information on events and there is a wide concern about the value of their data for engineering decision making. Data should be collected under strict rules, defining components, failure and individual maintenance actions accurately. As this requires a lot of effort, one should concentrate on the most relevant areas. Identifying these is also a complex problem. Exceptions are the condition monitoring programs, for which usually dedicated manpower is available.

An alternative to statistical data analysis, is to elicit information on failure distributions from maintenance technicians. Although it is far more flexible, it is not easy either and may seem subjective; a formal method to overcome this is given in Van Noortwijk et al. (1992). One should realise that under good maintenance practices there are little failures. If failures occur repeatedly, one may either change the system design or its operation to prevent failures, with the result that data collection has to start again. Other changes to systems have the same effect.

Apart from problems with deterioration and failure data, one also has problems with cost data, especially with the indirect costs. It is easy to calculate direct maintenance costs, but far more difficult to quantify the benefits of maintenance. In case of maintenance for production equipment one has to value the increase in reliability, availability or efficiency. The latter may be the easiest to judge. The first two are more difficult, especially if the equipment bears a complex relation with the final products le.g. one may use other equipment, or equipment is not continuously needed). If maintenance benefits are for another department than the one paying, one has even more problems. In cases where extending system life is the maintenance objective (e.g. roads) one may set norms or ask economists to do a specific calculation. 
In all cases, decision support systems can help in reducing uncertainty on data. By experimenting with different values one gets an impression on the importance of data, which then helps in deciding whether more time should be spend on getting better data.

\section{Future prospects}

Computers are becoming cheaper and more powerful. Moreover, improvements in software technology allow faster and better software development, with more intelligence embedded. Besides, it is widely accepted that the amount of capital invested in technical systems will continue growing. Due to increased demands on performance of these systems (with respect to quality, reliability and safety), spending on maintenance is likely to grow as well, a trend which is visible in many companies. Contracting out maintenance further increases the need for a higher quality of decision making. Maintenance optimisation models, embedded in decision support systems provide an objective and quantitative way of decision making. One which is defendable, and therefore very-well suited for group decision making, especially since it allows the evaluation of the economic consequences of decisions. As the models constitute the only approach which combines reliability with economics, they are in fact nothing more than extensions of the basic economic methods to justify maintenance. In many cases it is not the outcomes of the models which has most value, but the structuring of the decision process, the reduction of uncertainty and the better insight into of the effects of decisions across maintenance and production areas, which yield most benefits.

\section{Conclusions}

The impact of maintenance optimisation on decision making within maintenance organisations has been limited so far, but the few cases do show that considerable cost savings can be obtained. As application tool 
technology (both soft- and hardware) is only recently available against reasonable costs and is rapidly developing, we expect that the future will see much more maintenance decision support systems incorporating optimisation models, though several hurdles still need to be taken.

\section{Acknowledgement}

The author would like to acknowledge various people, especially Adriaan Smit and Cyp van Rijn from the Koninklijke/Shell-Laboratorium, Amsterdam for their contribution to the analysis reported in this paper.

\section{References}

Both, H. (1989), "Maintenance and Failure Behaviour of Technical Systems: Design and Applications of a Simulation Model", Ph.D. thesis, (in Dutch), Univ. of Eindhoven, NL

Dekker, R., (1992a), "Applications of Maintenance Optimisation Models: a Review and Analysis", Report Econometric Inst. 9228/A, Erasmus University Rotterdam.

Dekker, R., (1992b), "A General Framework for Optimisation, Priority Setting, Planning and Combining Maintenance Activities", Report Econometric Inst. 9270/A, Erasmus Univ. Rotterdam.

Dekker, R., Smit, A.C.J.M. and Van Rijn, C.F.H., (1993), "Maintenance Optimisation Models and their Application in Practice" (in Dutch), Doelm. Bedrijfsv., 5, nr. 1, p.4-7.

Golabi, K., Kulkarni, R.B. and Way, G.B., (1982), "A Statewide Pavement Management System", Interf. 12, p. 5-21.

Hontelez, J.A.M. (1992), "OPTIMON - User manual version 2" (in Dutch), Report FEL-92-C273, FEL-TNO, Den Haag.

Kralj, B.L. and Petrovic, R., (1987), "Optimal Preventive Maintenance Scheduling of Thermal Generating Units in Power Systems - A Survey of Problem Formulations and Solution Methods", Eur. Journ. Oper. Res., 35, p. 1-15.

Sherif, Y.S., (1982), "Reliability Analysis: Optimal Inspection and Maintenance Schedules of Failing Systems", Micr. Electr. \& Reliab. 22, p.59-115. 
Van Bommel P. and Roes, A.W., (1986), "What is OR doing between the Tracks", in: Tilanus, C.B., de Gans, O.B. and Lenstra, J.K. (eds.), "Quantitative Methods in Management: Case Studies of Failures and Successes", Wiley, Chichester, UK.

Van Gestel, P.J., (1990), "KMOSS, a Maintenance Optimization Support System", Proc. Scand. SRE Symposium, Studsvik, Sweden.

Van Noortwijk, J.M., Dekker, R., Cooke, R.M. and Mazzuchi, T.A., (1992), "Expert Judgment in Maintenance Optimization", IEEE Trans. on Rel., 41, p. 427-432.

Worms, J. and Van Harten, A., (1992), "Model based Decision Support for Planning of Road Maintenancen, working paper Univ. of Twente, (submitted for publication).

Woodhouse, J., (1986), "Relating Maintenance to Production and Company Profits", 6th. Nat. Conf. on Comp. for Maint. Mgmt. 